\title{
STYLISTIC STUDY OF THE ENGLISH TEXTBOOKS OF SECONDARY SCHOOLS OF GUJARAT: A COMPARATIVE STUDY
}

\author{
Ms. Reena K. Vihol \\ Dr. Dhaval R. Patel
}

\begin{abstract}
The purpose of this study is to analyse stylistic study of English textbooks of secondary schools of Gujarat. The researcher studied Grade IX and X textbooks to compare stylistic study with chosen parameters: Phonological aspect, Lexical aspect, and Syntactical aspect with randomly selected parameters.
\end{abstract}

Key Words: English Language, Stylistic Study, Analysis, Secondary School, English Textbooks, Phonological, Lexical, and Syntactical Aspect

\section{Introduction}

Language developed in various forms. Stylistic Study in literature and linguistics. The researcher investigated linguistics. Stylistics is the scientific study of style. In other words, literary text's structure of linguistic, Such as Phonological Aspect, Lexical Aspect and Syntactical Aspect. The variation in stylistics is because of the foremost influences of linguistics and literary that affects the meaning of a sound.

In this paper, the researcher studied a comparative study of "Stylistic Study of The English Textbooks of Secondary Schools of Gujarat" Grade IX and X. Mainly there are three parameters Phonological aspect, Lexical aspect, and Syntactical aspect selected. These parameters comprise with selected topics such as: Phonological aspect: Alliteration, Repetition, Assonance, Anaphora, Metaphor, Sound Elision or Contracted forms, Simile, Onomatopoeia, personification. Lexical Aspect: Affix, Collocation, Compound words, Indian words, Clipping words, Acronyms and Abbreviation and Syntactic Aspect: Capitalization, Italicization, Hyphenation, Spacing, Indention and Parentheses. The researcher studied each lesson and poem to analyse stylistic study.

\section{Gujarat}

\section{Grade IX}

\section{Phonological Aspects}

Alliteration 
e.g.

- "Green grass and tasty grains" (Pethani 36)

- "The flight be fleet" (Pethani 55)

- "Good bye and good night" (Pethani 66)

- "if you can't be a bush be a bit of the grass." (Pethani 120)

\section{Repetition}

e.g.

- "She cried and cried" (Pethani 2)

- "brighter and brighter" (Pethani 51)

- "May there be peace in heaven, peace in space, peace on earth." (Pethani 102)

\section{Assonance}

e.g.

'breeze', 'glee', 'green meadows', 'smooth'

"River, river! brimming river!" (Pethani 118)

\section{Anaphora}

e.g.

- "As we rush, as we rush in the train." (Pethani 55)

- "If you can't be a highway then just be a trail, If you can't be the sun be a star;" (Pethani 120)

\section{Metaphor}

e.g.

- "Be a bush if you can't be a tree" (Pethani 120)

- "If you can't be the sun be a star;" (Pethani 120)

\section{Sound Elision or Contracted forms}

e.g.

- “It's ok, dear." (Pethani 1)

- "I'm far stronger than you" (Pethani 51)

- "I'm sure you'll be able to get rid of this habit" (Pethani 66)

\section{Simile}

e.g.

- "As warm as a summer day." (Pethani 51)

- "Like impetuous youth" (Pethani 118)

- "Like eternity" (Pethani 118)

\section{Onomatopoeia}

e.g. 
'louder', 'brawling', 'leaping'

\section{Personification}

e.g.

- "River like a child at play" (Pethani 118)

- "A sea breathes in and out" (Pethani 122)

\section{Lexical Aspect}

Affix

e.g.

'Impetuous', 'Impossible', 'Misdeed', 'Unfortunately'

\section{Collocation}

e.g.

'To take a risk', 'Make progress', 'To save time', 'Come out'

\section{Compound words}

e.g.

'break-fast', 'news-paper', 'prayer-room', 'class-room'

\section{Indian words}

e.g.

"Indian Veena", "Ragas”, "Rani Ki Vaav", “Apsaras", "Patola”, “Ahimsa”

\section{Clipping words}

e.g.

- Ad: Advertisement

- Exam: Examination

- Gym: Gymnasium

\section{Acronyms and Abbreviation}

e.g.

- ATM: Automated Teller Machine

- SMS: Short Message Service

- UNESCO: United Nations Educational, Scientific and Cultural Organization

- ASI: Archaeological Survey of India

\section{Syntactic Aspect}

\section{Capitalization}


e.g.

'Queen Udayamati', 'Rules of My Room', 'Nanda Devi and Valley of Flowers Nation Park' 'T.V.', 'AIDS', 'HIV', 'A.D.'

\section{Italicization}

e.g.

Ahimsa (Pethani 102)

Mahakavi Kalidas, Abhijnan Shakuntalam (Pethani 108)

\section{Hyphenation}

e.g.

Emotion-word, fire-fighter, fire-station, Mitra-the bird, co-living, no-war

\section{Spacing}

e.g.

"Hey Abbu Miyan! Looking sad? Why are you sitting alone today? Where is your darling kalua?" (Pethani 36)

\section{Indention}

e.g.

"It was a cold morning and the girl had a shawl across her shoulder. Her feet were bare and her clothes were old. She was a young girl, walking gracefully towards me.

When she came to my window, she stopped....." (Pethani 56)

\section{Parentheses}

- "Sonia (opening her mouth a little bit, slowly)" (Pethani 16)

- "Mohan's brother, sister and nephews (who were also performing artists)" (Pethani 27)

- Angiosperms (Magnoliya, a flower plant)" (Pethani 112)

\section{Grade X}

\section{Phonological Aspects}

\section{Alliteration}

e.g.

- "Counsel Goel smiled softly staring at Prem Chopra." (Pethani 17)

- "My daughter little dear daughter" (Pethani 139)

- "A blue fish flying on the forehead of the cloud" (Pethani 139) 
- “I sail my streams..." (Pethani 141)

\section{Repetition}

e.g.

- “Chakkiben, Chakkiben Mari Sathe Ramva Aavso Ke Nahi, Aavso Ken Ahi?” (Pethani 96)

- "O my Luve is like a red, red rose" (Pethani 124)

\section{Assonance}

e.g.

'Carcasses', 'zoology', 'egg'

\section{Anaphora}

e.g.

- "If Kach dies,” said Devyani, “I will not live, and if you die, I also die.” (Pethani 82)

- "Till all the seas go dry.

Till all the seas go dry, my dear.” (Pethani 124)

- "And fare thee well, my only luve!

And fare thee well awhile!" (Pethani 124)

\section{Metaphor}

e.g.

- "NGO (EFL) beginning with the Turtle walk." (Pethani 29)

- "A teacher is a friend" (Pethani 66)

\section{Sound Elision or Contracted forms}

e.g.

- “wonderful idea, isn't it?" (Pethani 28)

- “No, I haven't, Mummy. (Pethani 73)

- “Smritiben: I'm sorry, she isn't in. who's speaking?" (Pethani 89)

\section{Simile}

e.g.

- "O my Luve is like the melody" (Pethani 124)

- "This song of mine will touch your forehead like a kiss of blessing" (Pethani 137)

\section{Onomatopoeia}

e.g.

'Blast', 'cracker', 'loud', 'bang', 'buzz', 'crackle' 
Towards Excellence: An Indexed, Refereed \& Peer Reviewed Journal of Higher Education / Ms. Reena Vihol \& Dr. Dhaval Patel/ Page 527-536

\section{Personification}

e.g.

"If Ram Singh 070, my robot, can shop for groceries, then why not for better things like jewels" (Pethani 16)

\section{Lexical Aspect}

\section{Affix}

e.g.

'Inefficient', Dislike, Lovable,

\section{Collocation}

e.g.

'Good Enough', 'Crystal Clear', 'Best Possible’

\section{Compound words}

'Fire-works', 'court-room', 'well-done'

\section{Indian words}

e.g.

'Vahugelo', 'Sughari', 'Vaiya', 'Gao', 'Mohalla'

\section{Clipping words}

e.g.

- Memo.: Memorandum

- Vet.: Veterinarian

- Gas.: Gasoline

\section{Acronyms and Abbreviation}

e.g.

- MGP: Mera Gao Power

- LED: Light Emitting Diode

- RTI: Right to Information

- CCTV: Closed Circuit Television

\section{Syntactic Aspect}

\section{Capitalization}

e.g.

- 'Mountain Man', “SYSTEM DISINTIGRATED” (Pethani 18)

- 'I LOVE YOU, MY TEACHER' (Pethani 66) 
- CFL, NGO', 'ISRO'

\section{Italicization}

e.g.

- 'Mother of Orphans' (Pethani 28)

- "I am there for all those who have no one" (Pethani 28)

- "Sanjivani Vidya" (Pethani 79)

\section{Hyphenation}

e.g.

- 'well-read' (Pethani 3)

- 'dome-shaped' (Pethani 16)

- 'pyrotechnics'-from the Greek word 'Pyr' (Pethani 55)

- 'Aaloo-poha' (Pethani 63)

\section{Spacing}

e.g.

"It is something unique. Maybe it is for pain, for joy, pride, disappointment, loneliness" the lord explained." (Pethani 42)

\section{Indention}

"with care and consideration, we can make our favourite festival a much safer one." (Pethani 57)

\section{Parentheses}

e.g.

- "Saputara which is on a plateau of Western Ghats (Sahyadri range), is a famous hill station in the dang district of Gujarat." (Pethani 9)

- "she worked for a man (the man used to be an athlete)" (Pethani 103)

- "Javed Habib's (famous hairstylist) life. (Pethani 74) 


\section{Table 1}

Percentage and Average of Stylistic Study

\begin{tabular}{|c|c|c|c|c|c|c|c|c|c|c|c|c|c|c|c|c|c|c|c|c|c|}
\hline \multicolumn{22}{|c|}{ Stylistic study } \\
\hline \multirow[b]{2}{*}{$\begin{array}{l}\text { Gujarat } \\
\text { State }\end{array}$} & \multicolumn{9}{|c|}{ Phonological Aspect } & \multicolumn{6}{|c|}{ Lexical Aspect } & \multicolumn{6}{|c|}{ Syntactical Aspect } \\
\hline & $\begin{array}{l}\text { All } \\
\text { iter } \\
\text { ati } \\
\text { on }\end{array}$ & $\begin{array}{l}\text { Re } \\
\text { pet } \\
\text { iti } \\
\text { on }\end{array}$ & $\begin{array}{l}\text { Asso } \\
\text { nanc } \\
\text { e }\end{array}$ & $\begin{array}{l}\text { Ana } \\
\text { phor } \\
\text { a }\end{array}$ & $\begin{array}{l}\text { Met } \\
\text { aph } \\
\text { or }\end{array}$ & $\begin{array}{l}\text { Sound } \\
\text { Elisio } \\
\mathrm{n} \text { or } \\
\text { Contr } \\
\text { acted } \\
\text { Forms }\end{array}$ & $\begin{array}{l}\text { Simi } \\
\text { le }\end{array}$ & $\begin{array}{l}\text { Ono } \\
\text { mato } \\
\text { poei } \\
\text { a }\end{array}$ & $\begin{array}{l}\text { Pers } \\
\text { onifi } \\
\text { catio } \\
\mathrm{n}\end{array}$ & $\begin{array}{l}\text { Affi } \\
x\end{array}$ & $\begin{array}{l}\text { Col } \\
\text { loc } \\
\text { ati } \\
\text { on }\end{array}$ & $\begin{array}{l}\text { Com } \\
\text { poun } \\
\text { d } \\
\text { Wor } \\
\text { ds }\end{array}$ & $\begin{array}{l}\text { India } \\
\mathrm{n} \\
\text { Wor } \\
\text { ds }\end{array}$ & $\begin{array}{l}\text { Cli } \\
\text { ppi } \\
\text { ng } \\
\text { W } \\
\text { ord } \\
\text { s }\end{array}$ & $\begin{array}{l}\text { Acron } \\
\text { yms } \\
\text { and } \\
\text { Abbre } \\
\text { viatio } \\
\text { ns }\end{array}$ & $\begin{array}{l}\text { Capita } \\
\text { lizatio } \\
\mathrm{n}\end{array}$ & $\begin{array}{l}\text { Italici } \\
\text { zation }\end{array}$ & $\begin{array}{l}\text { Hyp } \\
\text { hen } \\
\text { atio } \\
n\end{array}$ & $\begin{array}{l}\text { Spa } \\
\text { cin } \\
\mathrm{g}\end{array}$ & $\begin{array}{l}\text { Inde } \\
\text { ntio } \\
n\end{array}$ & $\begin{array}{l}\text { Pare } \\
\text { nthes } \\
\text { es }\end{array}$ \\
\hline IX & $\begin{array}{l}10 \\
0\end{array}$ & \begin{tabular}{|l}
10 \\
0
\end{tabular} & 100 & 100 & 100 & 100 & 100 & 100 & 100 & 100 & $\begin{array}{l}10 \\
0\end{array}$ & 100 & 100 & $\begin{array}{l}10 \\
0\end{array}$ & 100 & 100 & 100 & 100 & 100 & 100 & 100 \\
\hline$X$ & $\begin{array}{l}10 \\
0\end{array}$ & \begin{tabular}{|l|}
10 \\
0 \\
\end{tabular} & 100 & 100 & 100 & 100 & 100 & 100 & 100 & 100 & $\begin{array}{l}10 \\
0\end{array}$ & 100 & 100 & $\begin{array}{l}10 \\
0\end{array}$ & 100 & 100 & 100 & 100 & 100 & 100 & 100 \\
\hline Ave. & $\begin{array}{l}10 \\
0\end{array}$ & \begin{tabular}{|l}
10 \\
0
\end{tabular} & 100 & 100 & 100 & 100 & 100 & 100 & 100 & 100 & $\begin{array}{l}10 \\
0\end{array}$ & 100 & 100 & $\begin{array}{l}10 \\
0\end{array}$ & 100 & 100 & 100 & 100 & 100 & 100 & 100 \\
\hline
\end{tabular}




\section{Conclusion}

In this paper, the research studied each lesson and poem to analyse the stylistic study. both the textbooks of secondary schools of Gujarat grade IX and X covered all the parameters of stylistic study. And both the textbooks found equal outcomes. 


\section{Works Cited}

Chavda, H.N., et al. English (S.L), Std. 9. First Edition:2016 ed., Gandhinagar, Director, Gujarat State Board of School Textbooks, "Vidyayan", Sector 10-A, p. 130. 2019. print.

Chavda, H.N., et al. English (S.L), Std. 10. First Edition:2017 ed., Gandhinagar, H.N. Chavda, Director, on behalf of Gujarat State Board of School Textbooks, "Vidyayan", Sector 10-A, p. 146. 2019. print

Modern Language Association. MLA Handbook for Writers of Research Papers. $7^{\text {th }}$ ed. New Delhi: East-West Press Pvt Ltd., 2009. Print.

“What Is Stylistic Study in English Language - Bing." Www.bing.com, Web.19 April, 2021.

\section{Ms. Reena K. Vihol}

Research Scholar

Gujarat University, Ahmedabad

\&

Dr. Dhaval R. Patel

Associate Professor

Vision College of Education

Navrangpura, Ahmedabad 